\title{
Situação epidemiológica da brucelose bovina no Estado do Mato Grosso do Sul
}

[Epidemiological situation of bovine brucellosis in the State of Mato Grosso do Sul, Brazil]

\author{
S.C. Chate ${ }^{1}$, R.A. Dias ${ }^{1}$, M. Amaku ${ }^{1}$, F. Ferreira ${ }^{1}$, G.M. Moraes $^{2}$, A.A. Costa Neto ${ }^{3}$, L.A.R.C. Monteiro ${ }^{3}$, \\ J.R. Lôbo $^{2}$, V.C.F. Figueiredo ${ }^{2}$, V.S.P. Gonçalves ${ }^{4}$, J.S. Ferreira Neto ${ }^{1 *}$ \\ ${ }^{1}$ Faculdade de Medicina Veterinária e Zootecnia - USP \\ Av. Prof. Dr. Orlando Marques de Paiva, 87 \\ 05508-270 - São Paulo, SP \\ ${ }^{2}$ Departamento de Saúde Animal SDA-MAPA - Brasília, DF \\ ${ }^{3}$ Agência Estadual de Defesa Sanitária Animal e Vegetal - IAGRO - Campo Grande, MS \\ ${ }^{4}$ Faculdade de Agronomia e Medicina Veterinária - UnB - Brasília, DF
}

\begin{abstract}
RESUMO
Realizou-se um estudo para caracterizar a situação epidemiológica da brucelose bovina no Estado de Mato Grosso do Sul. Foram definidos três estratos (regiões): Pantanal-corte, Planalto-corte e Planaltoleite, este último subdividido em Bolsão, Campo Grande e Dourados. Em cada estrato foram amostradas aleatoriamente propriedades e, dentro dessas, foi escolhido, de forma aleatória, um número préestabelecido de animais, dos quais foi obtida uma amostra de sangue. No total, foram amostrados 14.849 animais, provenientes de 1.004 propriedades. Em cada propriedade amostrada foi aplicado um questionário epidemiológico para verificar suas características e também para detectar transtornos reprodutivos que poderiam estar associados à infecção brucélica. O teste utilizado foi o do antígeno acidificado tamponado. $\mathrm{O}$ rebanho foi considerado positivo se pelo menos um animal foi reagente à prova sorológica. Para o Estado, a prevalência de focos foi de 41,5\% [36,5-44,7\%]. As prevalências de focos e de animais infectados por estrato foram, respectivamente, de: 59,0\% [52,8-64,9\%] e 12,6\% [9,1-17,2\%] para o estrato Pantanal-corte, e 40,6\% [35,8-45,5\%] e 4,5\% [2,1-9,0\%] para Planalto-corte. No estrato Planalto-leite, a prevalência de focos foi de 33,1\% [28,4-38,1\%]. Os fatores de risco (odds ratios, OR) associados à condição de foco foram: ter $\geq 500$ vacas $(\mathrm{OR}=2,46[1,81-3,34])$, ocorrência de bezerros fracos $(\mathrm{OR}=1,20[0,87-1,65])$ e uso da inseminação artificial $(\mathrm{OR}=0,71[0,50-1,01])$.
\end{abstract}

Palavras-chave: bovino, brucelose, prevalência, Mato Grosso do Sul

\begin{abstract}
A study to characterize the epidemiological status of bovine brucellosis in the State of Mato Grosso do Sul was carried out. The State was divided in three regions: beef cattle Pantanal, beef cattle Plateau, and dairy cattle Plateau. Herds were randomly sampled in each region and a pre-established number of animals were sampled in each of these herds. A total of 14,849 serum samples from 1,004 herds were collected. In each herd, it was applied an epidemiological questionnaire focused on herd traits as well as clinical signs that could be associated with the brucellosis infection. The serum samples were tested for antibodies against Brucella spp. by the Rose-Bengal test. The herd was considered positive if at least one animal was positive. The prevalence of infected herds in the State was 41.5\% [36.5-44.7\%]. The prevalence of infected herds in the regions were, respectively: 40.6\% [35.8-45.5\%] for beef cattle Plateau, 59.0\% [52.8-64.9\%] for beef cattle Pantanal, and 33.1\% [28.4-38.1\%] for dairy cattle Plateau. The risk factors (odds ratio, OR) associated with the presence of the infection were: herd size larger than 500 cows $(O R=2.46$ [1.81-3.34]), birth of weak calf $(O R=1.20$ [0.87-1.65]), and use of artificial insemination $(O R=0.71[0.50-1.01])$.
\end{abstract}

Keywords: cattle, brucellosis, prevalence, Mato Grosso do Sul, Brazil

Recebido em 27 de março de 2009

Aceito em 23 de setembro de 2009

*Autor para correspondência (corresponding author)

E-mail: jsoares@vps.fmvz.usp.br 


\section{INTRODUÇÃO}

O Estado do Mato Grosso do Sul, criado em 1979 a partir da divisão do antigo Estado de Mato Grosso, está localizado na região CentroOeste e sua extensão territorial corresponde a $18 \%$ da região Centro-Oeste e $4,2 \%$ do Brasil, com $358.158,7 \mathrm{~km}^{2}$. O Pantanal sul-matogrossense ocupa uma área de $89.318 \mathrm{~km}^{2}$, correspondente a $25 \%$ do território estadual. O Estado é dividido em duas grandes bacias hidrográficas: a do Rio Paraná, constituída basicamente de chapadões, planaltos e vales, e a do Rio Paraguai, constituída de patamares, depressões e depressões inter-patamares, formando o Pantanal nas regiões chaquenha e pantaneira (Mato Grosso do Sul, 2007).

Faz fronteira ao sul com dois países: Bolívia e Paraguai. Com posição geográfica privilegiada, também faz divisa com cinco Estados brasileiros: Minas Gerais, São Paulo, Paraná, Goiás e Mato Grosso (Mato Grosso do Sul, 2007).

A vocação pecuária do Estado teve início com colonos espanhóis, que se instalaram no Pantanal sul-mato-grossense a partir de 1600. Mais tarde, com a fundação das missões jesuíticas do Itaim, novos rebanhos foram introduzidos (Esselin, 2003). Juntamente com a erva mate, a pecuária contribuiu para incorporar o Estado ao mercado nacional (Esselin, 2003).
O efetivo bovino do Estado do Mato Grosso do Sul é de 24,5 milhões, correspondente a $12 \%$ do rebanho brasileiro, que totaliza 207 milhões de cabeças (IBGE, 2007). O número total de propriedades é de 38.346, divididas entre as regiões do Pantanal e do Planalto. As propriedades da região do Pantanal, formada por oito municípios, são essencialmente de corte, enquanto na região do Planalto, formada por 71 municípios, existem propriedades de exploração de corte e de leite (bacias leiteiras do Bolsão de Campo Grande e de Dourados).

A Agência Estadual de Defesa Sanitária Animal e Vegetal do MS (IAGRO ) foi criada em 1978, após a implantação do Estado. Hoje dispõe de 80 escritórios locais, distribuídos por todos os municípios, 11 escritórios regionais e uma sede central. $\mathrm{O}$ combate à brucelose começou a se estruturar de forma mais organizada no Estado a partir de julho de 2002, com a implementação do programa de vacinação obrigatória definido no Programa Nacional de Controle e Erradicação da Brucelose e Tuberculose PNCEBT (Botene, 2008; IAGRO-MS; comunicação pessoal). Foram poucos os estudos de grande dimensão direcionados ao esclarecimento da situação epidemiológica da brucelose no Estado. Os principais foram sumarizados na Tab. 1.

Tabela 1. Principais estudos de prevalência de brucelose realizados no Estado do Mato Grosso do Sul (MS)

\begin{tabular}{|c|c|c|c|c|c|c|}
\hline $\begin{array}{l}\text { Ano da } \\
\text { coleta }\end{array}$ & Região do estudo & $\begin{array}{c}\text { Tipo de } \\
\text { exploração }\end{array}$ & $\begin{array}{c}\text { Teste } \\
\text { utilizado }\end{array}$ & $\begin{array}{l}\text { Planejamento } \\
\text { amostral }\end{array}$ & Prevalência & $\begin{array}{l}\text { Autores } \\
\text { (ano) }\end{array}$ \\
\hline 1975 & $\begin{array}{l}\text { Antigo Estado de } \\
\text { Mato Grosso }\end{array}$ & corte e leite & SARP & Houve & $\begin{array}{l}22,9 \% \text { de focos } \\
6,25 \% \text { de animais }\end{array}$ & Brasil (1977) \\
\hline 1982-1984 & Pantanal do MS & corte & AAT & Não informa & $10,19 \%$ de animais & $\begin{array}{l}\text { Cavalléro } \\
(1998)\end{array}$ \\
\hline 1994-1996 & Pantanal do MS & corte & $\mathrm{AAT}+2-\mathrm{ME}$ & Não houve & $3,43 \%$ de animais & $\begin{array}{l}\text { Pellegrin et } \\
\text { al. (1999) }\end{array}$ \\
\hline 2001 & MS & & AAT & Não houve & $8,6 \%$ de animais & $\begin{array}{l}\text { Almeida } \\
(2001)\end{array}$ \\
\hline 2003-2004 & Planalto do MS & corte e leite & $\mathrm{AAT}+2-\mathrm{ME}$ & Houve & $\begin{array}{l}37,3 \% \text { de focos } \\
5,6 \% \text { de animais }\end{array}$ & $\begin{array}{l}\text { Monteiro et } \\
\text { al. (2006) }\end{array}$ \\
\hline
\end{tabular}

Além desses estudos principais, existem três outras fontes com dados sobre a situação da brucelose bovina no Estado. A análise das notificações dos testes de soroaglutinação rápida em placa de 1981 a 1984, feita pela IAGRO, verificou 10.939 bovinos reagentes em 326.316 testados, o que resultou em frequência de 3,4\% (Lemos, 1998). Para o período de 1982 a 1984, o estudo da Universidade Federal de MS verificou 206 bovinos reagentes à sorologia para brucelose em 2.276 examinados, com frequência de 8,1\% (Lemos, 1998). Pellegrin et al. (1996) observaram soropositividade de 6,9\% em 198 touros e de $2 \%$ em 561 matrizes criadas na região do Pantanal sul-mato-grossense no período de 1994 a 1995. 
A partir de 2007, o Mato Grosso do Sul foi incluído no Projeto de Modernização da Defesa Agropecuária, conduzido pelo Ministério da Agricultura, Pecuária e Abastecimento (MAPA). Para isso, foi firmado o Convênio MA/SDA N. ${ }^{\circ}$ DFA/MS 003-00/97 que incluiu entre suas metas a realização de um grande estudo de prevalência da brucelose bovina no Estado. O estudo foi realizado em 1998, com metodologia bastante parecida com a proposta em 2003 para os demais estados da União, mas os resultados foram tratados de maneira bastante simples e utilizados para a produção de um relatório final de prestação de contas do mencionado Convênio. Assim, o Estado resolveu não realizar o novo estudo proposto em 2003, pois o de 1998 seria suficiente para se conhecer a situação epidemiológica da doença antes da implementação do PNCEBT. Isso faz sentido, pois a brucelose é uma doença crônica, de difusão lenta e, portanto, pouco suscetível a grandes modificações na sua distribuição e frequência no curto ou médio prazo. Então, optou-se por tomar os dados produzidos em 1998 e tratá-los conforme a metodologia do estudo de 2003, o que permitirá melhor implementação e gestão do PNCEBT em Mato Grosso do Sul.

\section{MATERIAL E MÉTODOS}

Tendo em vista o aproveitamento dos dados produzidos em 1998, os cálculos das prevalências nos animais para os circuitos leiteiros ficaram comprometidos pela impossibilidade de se ponderarem os resultados pelo número de animais existentes nas propriedades amostradas. Além disso, o estudo de fatores de risco não foi o mesmo, pois os questionários utilizados em 1998 e nos estudos realizados a partir de 2003 foram diferentes. Entretanto, os resultados produzidos revelaram de forma clara e objetiva a situação da brucelose bovina no Estado naquele ano.

O estudo foi planejado por técnicos do MAPA em colaboração com os da IAGRO. O trabalho de campo foi realizado por técnicos da IAGRO, no período de maio a outubro de 1998. Em cada propriedade amostrada, além da coleta de sangue para a sorologia, foi também aplicado um questionário epidemiológico, elaborado para obter informações sobre o tipo de exploração da propriedade e as práticas de manejo empregadas, de forma a permitir a realização do estudo de fatores de risco associados à presença da brucelose. $\mathrm{O}$ sangue foi coletado por punção da veia jugular com agulha descartável estéril em tubo com vácuo, previamente identificado. Os soros, armazenados em microtubos de plástico, foram mantidos a $-20^{\circ} \mathrm{C}$ até o momento da realização dos testes. Os testes sorológicos foram realizados pela IAGRO, com o apoio da Universidade Federal de Mato Grosso do Sul. Todas as informações geradas pelo trabalho de campo e de laboratório foram inseridas em um banco de dados específico, utilizado nas análises epidemiológicas.

Para que fossem conhecidas as diferenças regionais nos parâmetros epidemiológicos da brucelose bovina, o Estado foi dividido em dois estratos, levando-se em consideração os diferentes sistemas de produção, práticas de manejo, finalidades de exploração, tamanho médio de rebanhos e sistemas de comercialização. A capacidade operacional do serviço veterinário oficial do Estado também foi levada em consideração para essa tarefa.

Em cada estrato, estimou-se a prevalência de propriedades infectadas pela brucelose bovina e de animais soropositivos por meio de um estudo amostral em dois estágios, dirigido para detectar focos da doença. No primeiro estágio, sorteou-se aleatoriamente um número pré-estabelecido de propriedades com atividade reprodutiva (unidades primárias de amostragem). No segundo, sorteou-se um número pré-estabelecido de fêmeas bovinas com idade igual ou superior a 24 meses (unidades secundárias de amostragem).

Nas propriedades rurais onde existia mais de um rebanho, foi escolhido o rebanho bovino de maior importância econômica, no qual os animais estavam submetidos às mesmas condições de manejo, ou seja, sob os mesmos fatores de risco. A escolha da unidade primária de amostragem foi aleatória, baseada no cadastro de propriedades rurais com atividade reprodutiva de bovinos. A propriedade sorteada que, por motivos vários, não pôde ser visitada, foi substituída por outra, nas proximidades, com as mesmas características de produção. O número de propriedades selecionadas por circuito foi estimado pela fórmula para amostras simples aleatórias (Thrusfield, 2007). Os parâmetros adotados para o cálculo foram: nível de 
confiança de 0,95 , prevalência estimada de 0,25 e erro de 0,05. A capacidade operacional e financeira do serviço veterinário oficial do Estado também foi levada em consideração para a determinação do tamanho da amostra por estrato.

O planejamento amostral para as unidades secundárias visou estimar um número mínimo de animais a ser examinado, dentro de cada propriedade, de forma a permitir a sua classificação como foco ou não foco de brucelose. Para tanto, foi utilizado o conceito de sensibilidade e especificidade agregadas. Para efeito dos cálculos foram adotados os valores de $95,0 \%$ e $99,0 \%$, respectivamente, para a sensibilidade e a especificidade do teste utilizado (Fletcher et al., 1998) e 20\% para a prevalência estimada. Nesse processo foi usado o programa Herdacc versão 3 e o tamanho da amostra escolhido foi aquele que permitiu valores de sensibilidade e especificidade de rebanho superiores a $85 \%$.

Operacionalmente, foram amostradas 15 fêmeas com idade igual ou superior a 24 meses por propriedade e, naquelas com menos de 15 fêmeas, a totalidade de animais. A escolha das fêmeas dentro das propriedades foi aleatória.

As amostras de soro foram submetidas ao teste do antígeno acidificado tamponado ou teste Rosa Bengala segundo Alton et al. (1988), e não foi realizado o teste confirmatório. Uma propriedade foi considerada positiva quando se detectou pelo menos um animal positivo.

A prevalência de focos de brucelose foi calculada para todo o Estado e também para cada estrato. Em função das informações disponíveis no banco de dados, só foi possível calcular a prevalência de animais para os estratos Planaltocorte e Pantanal-corte. A prevalência aparente e o respectivo intervalo de confiança foram calculados conforme Dean et al. (1994).

As prevalências de focos para todo o Estado e para os estratos foram calculadas de forma ponderada, conforme preconizado por Dohoo et al. (2003).
O peso de cada propriedade no cálculo da prevalência de focos para todo o Estado e para os estratos corte (total) e leite (total) foi dado por

$$
P_{1}=\frac{\text { propriedades no estrato/região }}{\text { propriedades amostradas no estrato/região }}
$$

O peso de cada animal no cálculo da prevalência de animais nos estratos Planalto-corte e Pantanalcorte foi dado por

$$
P_{2}=\frac{\text { fêmeas } \geq 24 \text { meses na propriedade }}{\text { fêmeas } \geq 24 \text { meses amostradas na propriedade }}
$$

As variáveis analisadas no estudo de fatores de risco foram: número de vacas com idade superior a 24 meses, uso de inseminação artificial e ocorrência de problemas reprodutivos (aborto, retenção de placenta, repetição de cio, nascimento de bezerros fracos e orquite).

As variáveis foram organizadas de modo a apresentarem-se em escala crescente de risco. Quando necessário, realizou-se a recategorização dessas variáveis. A categoria de menor risco foi considerada como base para a comparação das demais categorias. As variáveis quantitativas foram categorizadas em percentis.

Foi feita uma primeira análise exploratória dos dados (univariada) para seleção daquelas com $\mathrm{p} \leq 0,20$ para o teste do $\chi^{2}$ ou exato de Fisher e, subsequente, oferecimento dessas à regressão logística. Os cálculos foram realizados com o auxílio do programa SPSS, version 9.0.

\section{RESULTADOS E DISCUSSÃO}

O Estado foi dividido em dois diferentes estratos: corte e leite. $\mathrm{O}$ estrato corte foi subdividido em duas regiões, Pantanal e Planalto. Na região Planalto, foram evidenciadas três bacias leiteiras: Bolsão, Campo Grande e Dourados (Fig. 1 e 2). Os dados censitários tomados como base para os cálculos da amostra e de prevalência foram os mais atualizados e disponíveis à época do trabalho de campo, em 1998.

A Tab. 2 traz um resumo desses dados censitários e também da amostra estudada em cada um dos circuitos produtores. 


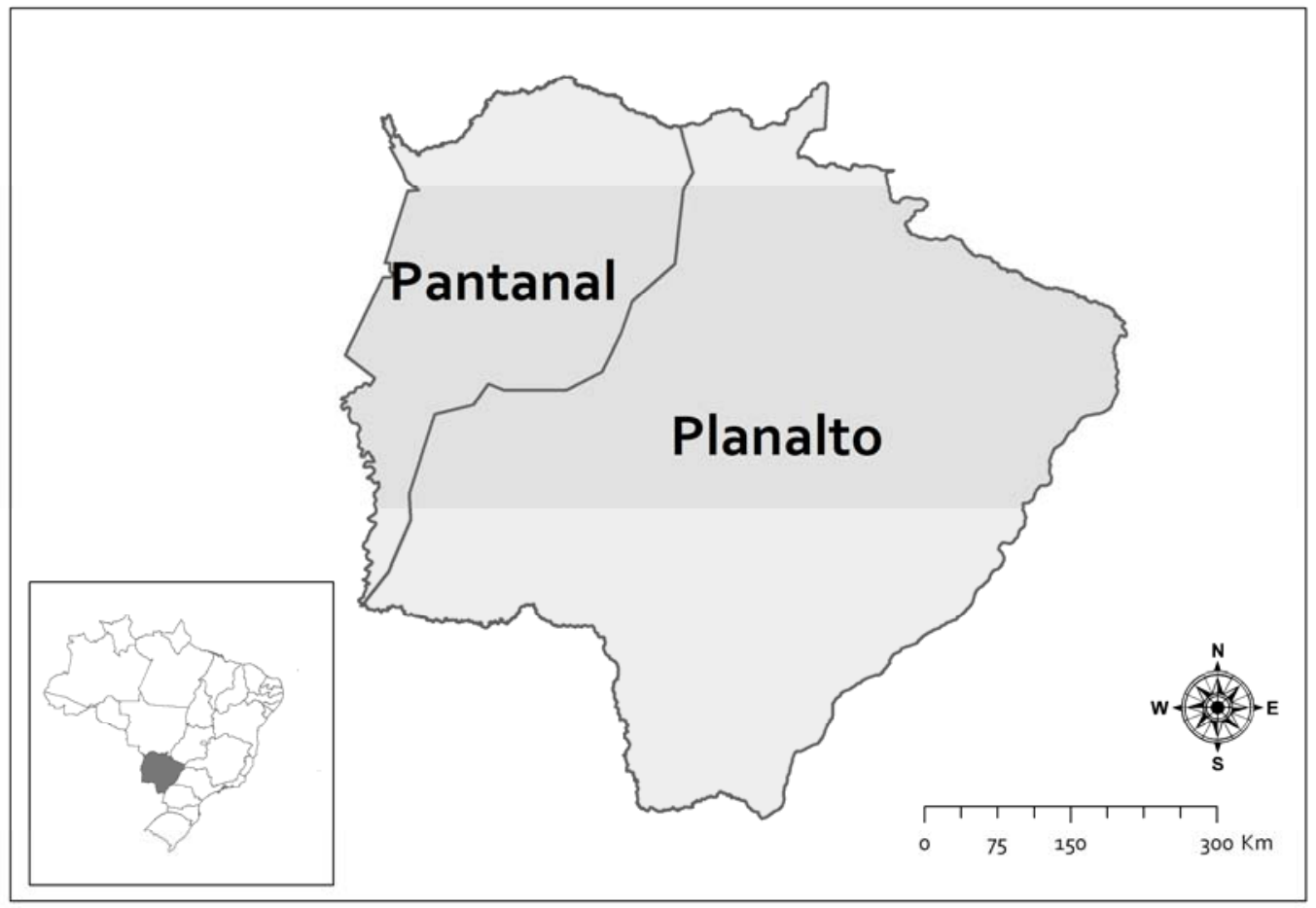

Figura 1. Mapa do Estado do Mato Grosso do Sul evidencia o estrato corte, dividido nas regiões Pantanal e Planalto.

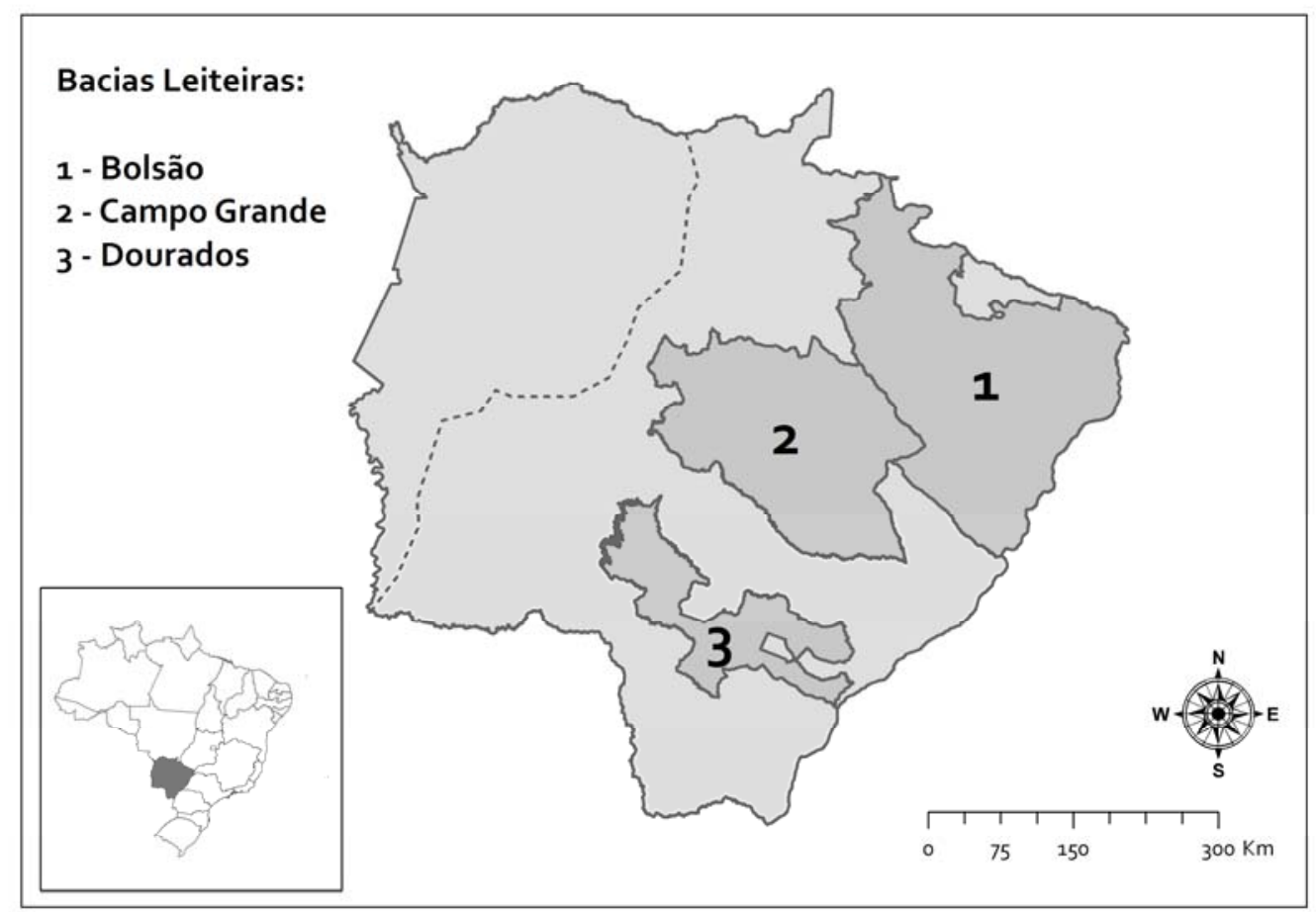

Figura 2. Mapa do Estado do Mato Grosso do Sul evidencia o estrato leite, dentro da região Planalto, composto pelas bacias leiteiras Bolsão, Campo Grande e Dourados. 
Tabela 2. Dados censitários da população bovina do Estado do Mato Grosso do Sul em 1998, segundo o estrato produtor

\begin{tabular}{|c|c|c|c|c|c|}
\hline \multirow{2}{*}{\multicolumn{2}{|c|}{ Estrato produtor }} & \multicolumn{2}{|c|}{$\begin{array}{c}\text { Propriedades com } \\
\text { atividade reprodutiva }\end{array}$} & \multicolumn{2}{|c|}{$\begin{array}{c}\text { Fêmeas com } \\
\text { idade } \geq 24 \text { meses }\end{array}$} \\
\hline & & total & amostradas & total & amostradas \\
\hline \multirow{3}{*}{ Corte } & Planalto & 37.652 & 387 & 5.276 .591 & 5.754 \\
\hline & Pantanal & 2.171 & 251 & 1.061 .994 & 3.712 \\
\hline & Total & 39.823 & 638 & 6.338 .585 & 9.466 \\
\hline \multirow{4}{*}{ Leite } & Bolsão & 1.902 & 129 & $*$ & 1.907 \\
\hline & Campo Grande & 1.205 & 84 & - & 1.237 \\
\hline & Dourados & 2.444 & 153 & - & 2.239 \\
\hline & Total & 5.551 & 366 & - & 5.383 \\
\hline \multicolumn{2}{|c|}{ Total geral } & 45.374 & 1.004 & - & 14.849 \\
\hline
\end{tabular}

*Dado indisponível. Fonte: IAGRO

Com base na amostra, foram calculadas as medianas do número de fêmeas com idade $\geq 24$ meses para os vários estratos. A mediana de matrizes no estrato Pantanal-corte foi de 400, no Planalto-corte 245 e no Leite, 55.

$\mathrm{Na}$ Tab. 3, são mostrados os resultados de prevalência de focos no Estado e nos estratos, e na Tab. 4, é mostrada a prevalência de animais segundo o estrato de gado de corte.

Na Tab. 5 mostram-se os resultados da análise univariada e na Tab. 6, o modelo final da regressão logística.

Tabela 3. Prevalência de focos de brucelose bovina nos estratos do Estado do Mato Grosso do Sul

\begin{tabular}{|c|c|c|c|c|c|}
\hline & & Prop & ades & & \\
\hline & & Tectodoc & Docitiva & & \\
\hline & Planalto & 387 & 157 & 40,6 & {$[35,8-45,5]$} \\
\hline & Pantanal & 251 & 148 & 59,0 & {$[52,8-64,9]$} \\
\hline & Total corte & 638 & 305 & 41,6 & {$[37,0-46,3]$} \\
\hline Leite & Bolsão & 129 & 37 & 28,7 & {$[21,5-37,1]$} \\
\hline & Campo Grande & 84 & 32 & 38,1 & {$[28,4-48,9]$} \\
\hline & Dourados & 153 & 52 & 34,0 & {$[26,9-41,9]$} \\
\hline & Total leite & 366 & 121 & 33,1 & {$[28,4-38,1]$} \\
\hline Total E & & 1.004 & 426 & 41,5 & {$[36,5-44,7]$} \\
\hline
\end{tabular}

IC: intervalo de confiança. 
Tabela 4. Prevalência de brucelose bovina no estrato corte no Estado do Mato Grosso do Sul

\begin{tabular}{llcccc} 
& \multirow{2}{*}{ Estrato } & \multicolumn{2}{c}{ Animais } & & $\begin{array}{c}\text { Prevalência } \\
(\%)\end{array}$ \\
\cline { 2 - 4 } & Pestados & Positivos & & IC (95\%) \\
\hline Corte & Planalto & 5.754 & 259 & 4,5 & {$[2,1-9,0]$} \\
& Pantanal & 3.712 & 468 & 12,6 & {$[9,1-17,2]$} \\
\hline
\end{tabular}

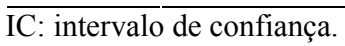

Tabela 5. Resultados da análise univariada dos possíveis fatores de risco associados à condição de foco de brucelose bovina no Estado do Mato Grosso do Sul

\begin{tabular}{lccc}
\hline \multicolumn{1}{c}{ Variável } & Expostos/casos & Expostos/controles & $\mathrm{p}$ \\
\hline Apresentou problema reprodutivo & $233 / 366$ & $317 / 502$ & 0,877 \\
Reportou ocorrência de aborto & $163 / 403$ & $209 / 544$ & 0,528 \\
Reportou ocorrência de retenção de placenta & $152 / 396$ & $220 / 527$ & 0,303 \\
Reportou ocorrência de repetição de cio & $149 / 383$ & $209 / 523$ & 0,748 \\
Reportou ocorrência de bezerros fracos & $97 / 391$ & $110 / 526$ & 0,163 \\
Reportou ocorrência de orquite & $8 / 390$ & $14 / 518$ & 0,527 \\
Usa inseminação artificial & $67 / 426$ & $113 / 578$ & 0,119 \\
Possui $\geq 500$ vacas & $144 / 426$ & $101 / 578$ & 0,001 \\
\hline
\end{tabular}

Tabela 6. Modelo final da regressão logística multivariada para os fatores de risco (odds ratio) associados à condição de foco de brucelose bovina no Estado do Mato Grosso do Sul

\begin{tabular}{lccc}
\hline \multicolumn{1}{c}{ Variável } & Odds ratio & IC (95\%) & $\mathrm{p}$ \\
\hline Rebanho com $\geq 500$ vacas & 2,46 & {$[1,81-3,34]$} & 0,001 \\
Uso de inseminação artificial & 0,71 & {$[0,50-1,01]$} & 0,056 \\
Ocorrência de bezerros fracos & 1,20 & {$[0,87-1,65]$} & 0,259 \\
\hline
\end{tabular}

IC: intervalo de confiança.

Verificou-se alta prevalência de focos (Tab. 3) com tendência de prevalências maiores nos estratos de corte. A prevalência de focos no estrato Pantanal-corte foi estatisticamente maior que a dos outros estratos. Neste estrato, a prevalência de animais foi mais alta que no estrato Planalto-corte (Tab. 4). É importante dizer que essa elevada prevalência de focos pode ser atribuída à estrutura de produção do Estado, organizado principalmente em grandes propriedades, com número elevado de matrizes. A mediana de matrizes no estrato Pantanal-corte foi de 400, no Planalto-corte, 245, e no Planaltoleite, 55. Nesse caso, existe uma tendência de a prevalência de focos resultar elevada, pois basta um animal positivo para classificar toda a unidade de produção como foco, ou seja, uma mesma prevalência de animais tende a determinar prevalência de focos mais elevada em regiões de propriedades grandes, com número elevado de animais, do que em regiões de propriedades pequenas com pequeno número de animais.

Nas três grandes bacias leiteiras do Estado (Tab. 3), observou-se prevalência semelhante de focos de brucelose, apesar de as estimativas pontuais mostrarem-se diferentes. Também não houve diferença entre a prevalência de focos dessas três regiões leiteiras e a verificada no estrato Planalto-corte, apesar de os números absolutos serem maiores para esse último.

Tratando-se de prevalência de focos, foi possível concluir que em 1998 existia forte tendência de maior concentração da doença nas propriedades de corte, com destaque para o estrato Pantanalcorte, e que a situação era homogênea nas três principais bacias leiteiras do Estado. Isso pode estar relacionado ao tamanho dos rebanhos de corte, grandes rebanhos significam intenso trânsito de animais, com subsequente aumento 
do risco de introdução e disseminação da doença, assim como, ao melhor trabalho desenvolvido pelas cooperativas leiteiras do Estado no sentido de combater os focos da doença (Poester et al., 2002).

Por tratar-se de Estado criado em 1979, a partir da divisão do antigo Estado de Mato Grosso, é importante verificar se a situação da brucelose nos dois Estados é semelhante. Os dados indicam que a situação é bastante parecida, pois a prevalência de focos no MS, em 1997, foi de 41,5\% (Tab. 3) e no MT, em 2003, 42,2\% (Mato Grosso, 2004). Feita essa ressalva, é possível comparar estudos realizados no antigo Estado do Mato Grosso com aqueles realizados no atual Estado do Mato Grosso do Sul.

O grande estudo realizado pelo MAPA em 1975 mostrou que a prevalência de focos no antigo Estado de Mato Grosso foi de 22,9\%, mais baixa que a verificada no presente, para o ano de 1997. Em que pese a diferença metodológica, tanto no planejamento amostral quanto nos protocolos de testes empregados nos dois estudos, é razoável supor que a prevalência realmente aumentou no Estado, pois o efetivo bovino cresceu cerca de 3,6 vezes no período, de seis para 21 milhões de cabeças (www.ibge.gov.br), com consequente aumento do trânsito de animais. Essa situação, somada à ausência de programa organizado de combate à brucelose, resultou invariavelmente na disseminação da doença. Em 1975, a prevalência de animais foi de 6,2\% (Brasil, 1977) e em 2001, 8,6\% (Almeida, 2001), sugerindo aumento da prevalência a despeito das diferenças metodológicas dos dois estudos.

Para a região do Pantanal, Cavalléro (1998) obteve prevalência de $10,2 \%$ de animais positivos no período de 1982 a 1984, muito diferente da encontrada em estudo subsequente, realizado por Pellegrin et al. (1999), em que a prevalência de animais foi de $3,4 \%$ para o período de 1994 a 1996. Essa diferença pode ser atribuída ao reduzido número de animais amostrados, 309, no estudo de 1999, em relação aos 1942 amostrados em 1998. A prevalência de animais encontrada no período de 1982 a 1984 $(10,2 \%)$ é semelhante à verificada pelo presente estudo, para o ano de 1998 (12,6\%; Tab. 3). Ao que parece, o pantanal mato-grossense está em situação de equilíbrio, no qual o número de animais que se infectam é igual ao número de animais infectados que morrem, pois desde 1982 a prevalência permanece praticamente a mesma.

Na região do Planalto, Monteiro et al. (2006), ao realizarem coletas de sangue entre 2003 e 2004 em amostra sem estratificação para corte e leite, estimaram a prevalência de focos em 37,3\%, valor intermediário às prevalências de focos de $33,1 \%$ e $40,6 \%$, estimadas, respectivamente, para as propriedades de leite e corte no presente estudo, em coletas de sangue realizadas no ano de 1997 (Tab. 2). As prevalências de animais encontradas pelos dois estudos também foram próximas: 4,5\% em 1997 para as propriedades de corte (Tab. 3) e 5,6\% em 2003/2004 para propriedades de corte ou leite (Monteiro et al., 2006). Em resumo, esses dados sugerem que a prevalência da brucelose em MS aumentou até por volta das décadas de 1980/1990 e, desde então, tem apresentado uma situação de relativo equilíbrio, com alta prevalência de focos.

A forma mais eficiente e econômica para o Estado diminuir a prevalência de brucelose é por meio da vacinação, anual, de no mínimo $80 \%$ de bezerras entre três a oito meses com a amostra B19.

A ocorrência de bezerros fracos, embora tenha apresentado $\mathrm{p}=0,25$, foi mantida no modelo final como variável de ajuste pela plausibilidade biológica e por apresentar odds ratio próximo ao limite de significância (IC 95\%: 0,87-1,65; Tab. 6). Assim, o modelo final da regressão logística mostrou que ter 500 ou mais vacas e que o nascimento de bezerros fracos foram fatores associados à condição de foco de brucelose bovina no Estado. $\mathrm{O}$ uso da inseminação artificial mostrou-se um fator protetor (Tab. 6).

A associação entre tamanho de rebanho e presença de brucelose já foi demonstrada (Nicoletti 1980; Salman e Meyer, 1984). Nos grandes rebanhos não há diferenças individuais quanto à suscetibilidade à doença, mas algumas características desses rebanhos podem facilitar a transmissão da brucelose, tais como: maior frequência de reposição de animais, maior número de problemas relacionados ao controle sanitário e à influência na dinâmica da doença (Crawford et al., 1990). Christie (1969) observou que o aumento do rebanho resulta em aumento da probabilidade de ocorrência e persistência da infecção, e no aumento da prevalência da doença 
e da dificuldade de erradicá-la. Assim, número elevado de animais no rebanho significa maior risco de introdução e disseminação da brucelose. Importante ressaltar que ter mais de 500 vacas no rebanho, praticamente significa ser propriedade de corte no MS, pois $96 \%(242 / 252)$ das propriedades com mais de 500 vacas eram de corte. Esses dados confirmam os resultados obtidos no estudo de prevalências, no qual foi verificada maior concentração da infecção nos estratos de propriedades de corte.

A associação nascimento de bezerros fracos com condição de foco de brucelose bovina já foi relatada por vários autores (Nicoletti, 1986; Samartino e Enright, 1993), pois as diferenças de manifestações clínicas são decorrentes do desenvolvimento de imunidade. Em animais brucélicos, o aborto é um acontecimento constante na primeira gestação. Contudo, em gestações subsequentes, o mais comum é o nascimento de animais fracos, pois menor número de placentomas é atingido e menor é o grau de lesão observado. Portanto, a diminuição da passagem de nutrientes e oxigênio não chega a causar a morte, mas compromete o desenvolvimento do feto, o que se traduz pelo nascimento de animais pequenos $\mathrm{e}$ subdesenvolvidos (Pellegrin et al., 2006). Assim, a ocorrência de nascimento de bezerros fracos é uma consequência da brucelose.

O uso da inseminação artificial mostrou odds ratio inferior a um e no limite da significância, informando que essa prática está associada à condição de livre de brucelose. Essa variável é frequentemente utilizada como indicador do nível de tecnificação da produção, significando capacidade de incorporação de tecnologias e, por consequência, de práticas sanitárias mais acuradas. Assim, esse resultado indica que propriedades mais bem estruturadas do ponto de vista zootécnico e sanitário têm menor chance de serem focos de brucelose.

Esses resultados sugerem que o fator mais importante para a disseminação da brucelose no Estado é a introdução de animais sem cuidados sanitários, que emergiu de forma indireta, pela interpretação da variável ter 500 ou mais vacas.

Recomenda-se: concentrar esforços na obtenção, todos os anos, de uma cobertura vacinal mínima de $80 \%$ de fêmeas entre três e oito meses de idade com a vacina B19; desencorajar a introdução de animais sem cuidados sanitários.

\section{AGRADECIMENTOS}

À FAPESP, ao CNPq, à IAGRO-MS e ao MAPA pelo apoio financeiro.

\section{REFERÊNCIAS BIBLIOGRÁFICAS}

ALMEIDA, R.C.F. Diagnóstico da brucelose e tuberculose bovina. 2001. 30f. Monografia (Graduação) - Universidade Federal de Mato Grosso do Sul, Campo Grande, MS.

ALTON, G.G.; JONES, L.M.; ANGUS, R.D. et al. Techniques for the brucellosis laboratory. Paris: Institut National de la Recherche Agronomique, 1988. 545p.

BRASIL. Ministério da Agricultura e Abastecimento. Diagnóstico de saúde animal, Brasília, 1977. 735p.

CAVALLÉRO, J.C.M. Enfermidades causadoras de aborto: Brucelose. In: LEMOS, R.A.A. (Ed.). Principais enfermidades de bovinos de corte do Mato Grosso do Sul: Reconhecimento e diagnóstico. Campo Grande: UFMS, 1998. 536p.

CRAWFORD, R.P.; HUBER, J.D.; ADAMS, B.S. Epidemiology and surveillance. In: NIELSEN, K.; DUNCAN, J.R. (Ed.). Animal brucellosis. Boca Raton: CRC Press, 1990.p.131151.

CHRISTIE, T.E. Eradication of brucellosis in northern Ireland: Field problems and experiences. Vet. Rec., v.85, p.268-269, 1969.

DEAN, A.G.; DEAN, J.A.; COLOMBIER, D. et al. Epi-Info, Version 6: A word processing database, and statistics program for epidemiology on microcomputers. Atlanta, CDC, 1994. 601p.

DOHOO, I.; MARTIN, W.; STRYHN, H. Veterinary epidemiologic research. Charlottetown, Canadá: Atlantic Veterinary College, 2003. 706p.

ESSELIN, P.M. A pecuária no processo de ocupação e desenvolvimento econômico do Pantanal sul-mato-grossense (1830-1910). 2003. 86f Tese (Doutorado) - Pontifícia Universidade Católica do Rio Grande do Sul, Porto Alegre, RS. 
FLETCHER, R.H.; FLETCHER, S.W.; WAGNER, E.H. Clinical epidemiology: The essentials. 2.ed. Baltimore: Williams \& Wilkins, 1998. 246p.

IBGE. Rio de Janeiro, 2007. Disponível em: $<$ http:www.sidra.ibge.gov.br>. Acessado em: 7 mai. 2007.

LEMOS, R.A.A. Principais enfermidades de bovinos de corte no Estado do Mato Grosso do Sul. Campo Grande: UFMS, 1998. p.408-441.

MATO GROSSO. Instituto de Defesa Agropecuária do Estado de Mato Grosso. Cuiabá, 2004. Disponível em:http: \www.indea.mt.gov.br/html/index.php. Acessado em: 7 mai. 2007.

MATO GROSSO DO SUL. Governo do Estado de Mato Grosso do Sul. Disponível em: http://www.ms.gov.br/index.php?inside $=1 \& \mathrm{tp}=3$ $\&$ comp $=1725 \&$ show $=949$ Acessado em 10 out. 2007.

MONTEIRO, L.A.R.C.; PELLEGRIN, A.O.; ISHIKAWA, M.M. et al. Investigação epidemiológica da brucelose bovina em um estrato do Estado de Mato Grosso do Sul. Pesq. Vet. Bras., v.26, p.217-222, 2006.

NICOLETTI, P. The epidemiology of bovine brucellosis. Adv. Vet. Sci. Comp. Med., v.24, p.69-98, 1980.

NICOLETTI, P. Effects of brucellosis on bovine reproductive efficiency. In: MORROW, D.A. Current therapy in theriogenology. 2.ed., 1986. p.271-274.
PELLEGRIN, A.O.; SERENO, J.R.B.; SILVA, R.A.M.S. et al. Doenças da reprodução diagnosticadas no pantanal mato-grossense e região do planalto: Resultados preliminares. ENCONTRO DE LABORATÓRIOS DE DIAGNÓSTICOS VETERINÁRIO DO CONE SUL, 1996, Campo Grande. Anais... Campo Grande: UFMS, 1996. p.68-72.

PELLEGRIN, A.O; $\quad$ LEITE, R.M.H.; GUIMARÃES, P.H.S. et al. Prevalência de brucelose bovina no pantanal mato-grossense. In: CONGRESSO BRASILEIRO DE MEDICINA VETERINÁRIA, 26., Campo Grande, 1999.

PELLEGRIN, A.O.; LEITE, R.M.H.; SERENO, J.R.B. et al. Brucelose bovina no pantanal sulmato-grossense: Dados preliminares. Com. Tec. Embrapa, n.58, p.1-4, 2006.

POESTER, F.P.; GONÇALVES, V.S.P.; LAGE, A.P. Brucellosis in Brazil. Vet. Microbiol., v.90, p.55-62, 2002.

SALMAN, M.D.; MEYER, M.E. Epidemiology of bovine brucellosis in the Mexicali Valley, México: Literature review of disease-associated factors. Am. J. Vet. Res., v.45, p.1557-1560, 1984.

SAMARTINO, L.E.; ENRIGHT, F.M. Pathogenesis of abortion of bovine brucellosis. Comp. Immunol. Microbiol. Infec. Dis., v.16, p.95-101, 1993.

THRUSFIELD, M. Veterinary epidemiology. 3.ed. Oxford: Blackwell Science, 2007. 610p. 